\title{
A Patient with Graves' Disease and Three Types of Thyroid Carcinoma
}

\author{
Vania dos Santos Nunes, ${ }^{1}$ José V. Tagliarini, ${ }^{2}$ Mariângela E.A. Marques, ${ }^{3}$ \\ Josué A. de Godoi, ${ }^{4}$ and Glaucia M.F.S. Mazeto ${ }^{1}$
}

\section{Dear Editor:}

The prevalence of epithelial cell thyroid cancer (TC) has been increasing in patients with Graves' disease (GD) (1). In addition, anaplastic TC (ATC) may arise in the setting of papillary TC (PTC). It is likely, however, that the patient we report here, who had GD and three types of TC, is very unusual.

A 71-year-old woman presented at another facility with tremor, hyperhidrosis, palpitations, weakness, reduced appetite, and recent $20-\mathrm{kg}$ weight loss. She had a 10-year history of hypertension. Hyperthyroidism was diagnosed, and methimazole, $40 \mathrm{mg}$ daily, was prescribed. Three years later symptoms of hyperthyroidism were present but milder. She was examined in our clinic and was noted to have one hard 2-cm nodule in the right thyroid lobe (RL) and one hard 3-cm nodule in the left thyroid lobe (LL). Prior ultrasonography (US) showed a solid, heterogeneous $2.02-\mathrm{cm}^{3}$ mass in the RL and a solid $16.43-\mathrm{cm}^{3}$ heterogeneous mass with microcalcifications in the LL. She was clinically euthyroid on $30 \mathrm{mg}$ methimazole daily.

Serum thyrotropin (TSH) was $0.029 \mathrm{mIU} / \mathrm{mL}(0.27-4.2)$, free thyroxine was $1.52 \mathrm{ng} / \mathrm{dL}(0.93-1.7)$, and TSH receptor antibodies were positive at $162 \mathrm{U} / \mathrm{L}(\mathrm{RV} \leq 11.0 \mathrm{U} / \mathrm{L})$. Technetium-pertechnetate scintigraph showed an enlarged thyroid with cold areas in both lobes. US fine-needle aspiration biopsy of both nodules revealed a follicular neoplasm in the RL and a Hurthle cell tumor in the LL. The patient underwent left loboisthmectomy; frozen-section analysis revealed Hurthle cell carcinoma, and a total thyroidectomy was performed shortly thereafter. Histopathological examination showed a $23.4-\mathrm{cm}^{3}$ Hurthle cell tumor in the LL. There was also a $1.3-\mathrm{cm}$ follicular carcinoma in the RL and adjacent to it a $0.4-\mathrm{cm}$ anaplastic carcinoma (Fig. 1). The anaplastic carcinoma contained anaplastic fusiform cells that were negative for thyroglobulin (Tg) and were intermingled with cell groups positive for $\mathrm{Tg}$ (Fig. 2).

The patient was treated with 5040 cGy radiotherapy to the cervical region and, 6 months later, with $280 \mathrm{mCi}$ of 131iodine $\left({ }^{131} \mathrm{I}\right)$. The posttreatment whole-body scan (WBS) showed only thyroid bed uptake. One year later, cervical and brain magnetic resonance imaging, chest tomography, and

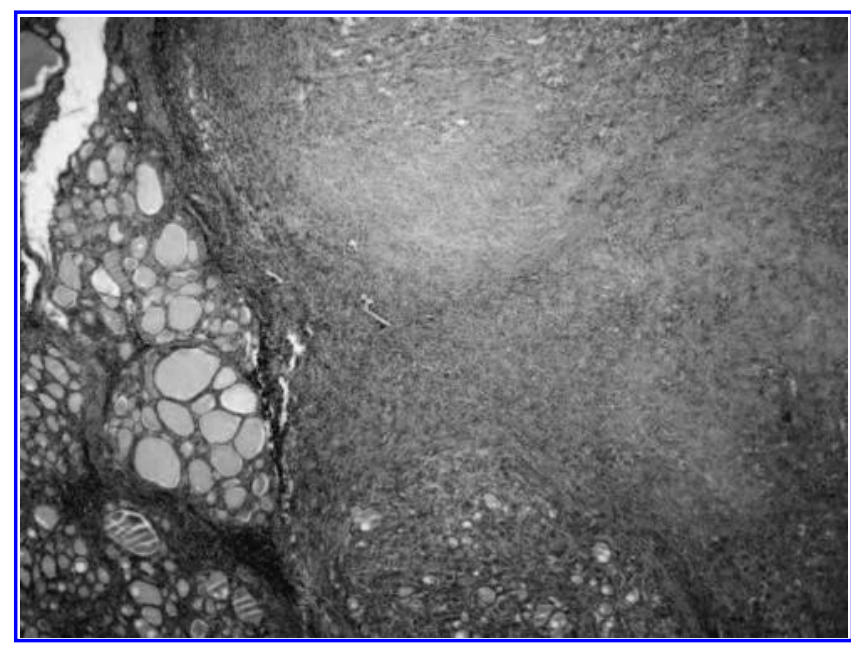

FIG. 1. Histologic section of the right lobe of the thyroid showing the transition from follicular carcinoma and anaplastic carcinoma. $H \& E, \times 200$.

abdominal US were negative, and serum $\mathrm{Tg}$ and anti-Tg antibodies were negative and undetectable. A WBS with thyroid hormone withdrawal performed 18 months after thyroidectomy was negative, but 1 month later the patient developed dyspnea, hip pain, and a left submandibular node. Chest tomography was consistent with pulmonary and left femur metastases. Fine-needle aspiration of the submandibular node and bronchial lavage cytology were suggestive of metastasis of anaplastic carcinoma. The patient died 2 months later.

The patient reported here is quite unusual not so much for some of her many associations, namely, GD with epithelial cell TC and PTC with ATC, but for the simultaneous combination of three types of TC and GD.

The coexistence of thyroid nodules and GD is widely described (2) and a number of studies have shown increased aggressiveness of TC in GD patients (3). Many investigators have strongly suggested that the thyroid-stimulating antibodies play a role in greater aggressiveness of thyroid

Departments of ${ }^{1}$ Internal Medicine, ${ }^{2}$ Ophtalmology and Otorhinolaryngology, and ${ }^{3}$ Pathology, Botucatu Medical School, São Paulo State University (UNESP), Botucatu, Brazil.

${ }^{4}$ School of Biological Sciences, University Center Nossa Senhora do Patrocinio (CEUNSP), Itu, Brazil. 




FIG. 2. Histologic section of the right lobe of the thyroid showing positive staining for thyroglobulin in the areas of follicular carcinoma and negative in anaplastic areas. H\&E, $\times 400$.

malignancy (4). These antibodies are closely linked to angiogenesis, which plays a crucial role in tumor growth and development. In addition, the genetic mutations involved in the TC may also have an important role in the carcinogenetic process, and together with the high level of circulating thyroidstimulating antibody may contribute to a more aggressive behavior of TC.

It is noteworthy that there was a sudden progression of ATC 1 month after the patient's last WBS, suggesting that, despite the background presence of TSH receptor antibodies, the rise in serum TSH levels associated with the prescan preparation may have stimulated the ATC. Although expression of TSH receptor in undifferentiated TC is often lost, residual expression of these receptors by the ATC tumor may, in part, explain the expansion of the tumor mass. Unfortunately, we were not able to study either expression of TSH receptors or the molecular aspects of her tumor tissue.

In summary, we present a remarkable patient with three types of TC and treated GD. The experience described in this case report is a reminder that cancer can occur in GD and that it may be associated with a worse prognosis. Therefore, we recommend that GD patients should undergo regular screening - both meticulous physical examination and ultrasonography-for early detection of TC.

\section{References}

1. Behar R, Arganini M, Wu TC, McCormick M, Straus FH 2nd, DeGroot LJ, Kaplan EL 1986 Graves' disease and thyroid cancer. Surgery 100:1121-1126.

2. Cantalamessa L, Baldini M, Orsatti A, Meroni L, Amodei V, Castagnone D 1999 Thyroid nodules in Graves' disease and the risk of thyroid carcinoma. Arch Intern Med 159:17051708.

3. Mazzaferi EL 1990 Thyroid cancer and Graves' disease. J Clin Endocrinol Metab 70:826-829.

4. Pellegriti G, Belfiore A, Giuffrida D, Lupo L, Vigneri R 1998 Outcome of differentiated thyroid cancer in Graves' patients. I Clin Endocrinol Metab 83:2805-2809.

Address correspondence to: Vania dos Santos Nunes, M.D. Departamento de Clínica Médica da Faculdade de Medicina de Botucatu Distrito de Rubião Junior, $s / n$ 18618-970 Botucatu-SP Brazil

E-mail: nunesvania2003@yahoo.com.br 